\title{
Sensitivity of Hartmannella (Acanthamoeba) to 5 -fluorocytosine, hydroxystilbamidine, and other substances
}

\author{
DAVID P. CASEMORE
}

From the Department of Virology, Public Health Laboratory, Middlesbrough, Teesside

SYNOPSIS The effect of 24 anti-amoebic and other chemotherapeutic compounds on six strains of hartmannellid amoebae was studied by tissue culture, agar diffusion, and liquid axenic culture.

Of the recognized anti-amoebic compounds only one inhibited growth of the amoebae, which were remarkable for their resistance.

Two compounds, hydroxystilbamidine isethionate and 5-fluorocytosine, showed some amoebicidal activity while some others were inhibitory.

Hartmannella and Naegleria have both been reported as causes of primary amoebic meningoencephalitis but recent reports on the role of normally saprophytic amoebae in human disease have tended to emphasize Naegleria as the chief potential pathogen (eg, Carter, 1968; Apley, Clarke, Roome, Sandry, Saygi, Silk, and Warhurst, 1970). However, the ability of some hartmannellae to grow at $33-37^{\circ} \mathrm{C}$, to produce degenerative changes in tissue culture, and to produce a lethal meningo-encephalitis in small laboratory mammals suggests that they should be regarded as facultatively pathogenic, as proposed by Griffin and others (Armed Forces Institute of Pathology, 1968).

The taxonomy of the hartmannellid group has not yet been resolved. Page (1967a and b, and personal communication) would give the amoebae used in this study the generic status Acanthamoeba: I do not feel competent to judge this point and have retained the nomenclature previously used. The potentially pathogenic nature of some hartmannellid amoebae suggested that an examination of their drug sensitivity would be worthwhile.

Received for publication 30 April 1970.
Materials and Methods

AMOEBAE

Six strains of amoebae were used. They conformed to Warhurst and Armstrong's description (1968) of Hartmannella castellanii. Their sources are shown in Table I.

\begin{tabular}{ll}
\hline Strain Number and Origin & Source \\
\hline 29 Manchester & Postmortem bronchial swab \\
324 Cirencester & Throat swab \\
B147 London & Throat swab \\
565 Leeds & Throat swab \\
524 Coventry & Throat swab \\
M67 Middlesbrough & Tissue culture contaminant
\end{tabular}

Table I Sources of amoebae and geographical origins

COMPOUNDS UNDER TEST

Compounds were chosen because they had reputed antiprotozoal activity, because they were chemically related to known antiprotozoal agents, because they were constituents of tissue-culture 
media that might be used for attempts at isolation of amoebae, or because their mode of action against other organisms suggested possible activity against amoebae.

Some compounds used for the treatment of entamoebiasis were not tested in this study in vitro because they depend for their activity on a chemical reaction within the intestinal tract, resulting in the local release of iodine, arsenic, etc.

Compounds were obtained as pure powders from the makers, or as pharmaceutical preparations for injection. Stock solutions were prepared, usually in distilled water, at a concentration of $1,000 \mu \mathrm{g} / \mathrm{ml}$. Each was tested for anti-amoebic activity at an arbitrary concentration of $100 \mu \mathrm{g} /$ ml. Compounds showing some effect at this concentration were tested by titration in at least two of the test systems used; those showing no effect or only slight inhibition at $100 \mu \mathrm{g} / \mathrm{ml}$ by all three methods were not tested further.

\section{TISSUE CULTURE}

HeLa cells were grown and maintained using conventional media and methods. Sensitivity tests were performed in media without any of the antibiotics normally used to control bacterial and mycotic growth.

\section{AGAR DIFFUSION}

Klebsiella-water-agar medium (Warhurst and Armstrong, 1968) was used; $1 \mathrm{ml}$ of the tenfold concentration of the compound being tested was mixed with $9 \mathrm{ml}$ of cooled, molten agar. Killed Klebsiella was used to avoid inconsistencies that might arise when the Klebsiella was itself inhibited by the compound under test.

\section{AXENIC CULTURE}

Several peptone media, with and without glucose, were tried (Neff, 1957; Adam, 1964). Best growth was obtained in a thin layer of medium containing $4 \%$ proteose-peptone (Difco) and $1 \%$ glucose, dissolved in tap water and sterilized by autoclaving (PPG medium).

In most cases a tenfold concentration of the compound was diluted with the medium and then inoculated with the amoebae. In some cases, however, the compound was added to a dense, actively growing culture.

Tests for combined drug action were performed using chess-board titrations to give varying concentration ratios in PPG medium in a divided Petri dish (Sneath and Stevens, 1967).

\section{INCUBATION}

All cultures were incubated at $37^{\circ} \mathrm{C}$. Proteosepeptone glucose and agar-culture containers were enclosed in polythene bags to reduce evaporation.
EXAMINATION

Although growth could often be detected by naked-eye examination, especially in PPG medium, all cultures were examined micro scopically after 24 hours and then at irregulaps intervals during the week after inoculation. Tests were controlled by inoculating each strain, in parallel with the test, into the same medium frees? from the compound under test. Results wer@ recorded as heavy, moderate, or scanty growth growth not detected, and, when marked differen=ces occurred, approximate proportions of cysts to trophozoites. Equivocal results, especially those involving few survivors after exposure to ins hibitory compounds, were checked by recoverin the amoebae and subculturing to assess theit viability.

\section{INTER PRETATION}

The relative amounts of growth recorded for tests and controls were interpreted as follows

\section{No effect}

When test and control showed no difference i rate of multiplication, yield of trophozoites, or rate of encystment.

\section{Inhibition without amoebicidal effect}

When any of the following effects were noted is test cultures: (a) slower rate of multiplication (b) lower yield of trophozoites, or (c) more rapi\$ encystment.

\section{Amoebicidal effect}

When (a) cysts recovered from (2) failed to vegetate in medium free from the compoun being tested, or (b) when the addition of a test compound to a dense, actively growing axenie culture of trophozoites caused loss of viability a? indicated by subculture of trophozoites to fres $\bar{b}$. medium free from the compound being tested.

\section{Results}

Table II shows the results of preliminary screening of the test substances or combinations at $\mathbb{2}$ concentration of $100 \mu \mathrm{g} / \mathrm{ml}$ of each compoundu Only four substances, amphotericin B, paromo? mycin, hydroxystilbamidine isethionate, anf 5-fluorocytosine, were sufficiently active in the screening tests to justify further studies. The purpose of the study was to investigate the sensi tivity pattern rather than to determine minimuri inhibitory concentrations (MICs). Although the strains of amoebae showed slight differences i\& their growth characteristics and MICs there was no difference in their sensitivity patterns, so drus effects on the six strains are not shown separatele

Antimetabolites acting on the folic acid cycle sulphonamides, trimethoprim, pyrimethamine 
tend to act synergistically. Different sulphonamides may vary in their efficacy to potentiate other substances acting on the folic acid cycle or in their suitability for use in this way. In any such combination the degree of potentiation may be affected by the ratio of compounds used. Chessboard titrations of trimethoprim/sulphamethoxazole and pyrimethamine/sulphormethoxine,

\begin{tabular}{ll}
\hline Compound & $\begin{array}{l}\text { Effect on Amoebae at } \\
100 \mathrm{\mu g} / \mathrm{ml}^{\mathbf{1}}\end{array}$ \\
\hline Acetarsol & No effect \\
Diloxanide furoate & No effect \\
Oxytetracycline & No effect \\
Benzyl penicillin & No effect \\
Streptomycin sulphate & No effect \\
Neomycin & No effect \\
Kanamycin & No effect \\
Paromomycin & I (marked) \\
Chloroquin & Morphological changes only \\
Nystatin & No effect \\
Metronidazole & VI \\
Polymyxin B & I (feeble) \\
Sulphonamides & VI \\
Trimethoprim lactate & No effect \\
Pyrimethamine sulphate & No effect \\
Trimethoprim + sulphamethoxazole I \\
Trimethoprim + polymyxin B & I \\
Pyrimethamine + sulphormethoxine I \\
Trimethoprim + pyrimethamine & I \\
Amphotericin B & I (marked) \\
Hydroxystilbamidine & AC \\
5-Fluorocytosine & AC \\
\hline
\end{tabular}

Table II Preliminary screening tests of 24 compounds alone or in combination

${ }^{1}$ In tests of two drugs together, each was used at a concentration of $100 \mu \mathrm{g} / \mathrm{ml}$.

I $=$ inhibition without amoebicidal effect

$\mathrm{AC}=$ amoebicidal

$\mathrm{VI}=$ variable inhibition reactions

- $=$ not tested

2Separate tests on sulphanilamide, sulphadiazine, sulphasomizole, sulphafurazole, sulphathiazole, sulphamethoxazole, and sulphormethoxine

\begin{tabular}{|c|c|c|c|}
\hline \multirow[t]{2}{*}{ Compound } & \multicolumn{3}{|c|}{$\begin{array}{l}\text { Effect on Amoebae at Concentration } \\
(\mu \mathrm{g} / \mathrm{ml})\end{array}$} \\
\hline & 100 & 10 & 1 \\
\hline Amphotericin B & $\begin{array}{l}\text { Marked } \\
\text { inhibition }\end{array}$ & Inhibition & $\begin{array}{l}\text { Feeble } \\
\text { inhibition }\end{array}$ \\
\hline Paromomycin & $\begin{array}{l}\text { Marked } \\
\text { inhibition }\end{array}$ & $\begin{array}{l}\text { Feeble } \\
\text { inhibition }\end{array}$ & No effect \\
\hline
\end{tabular}

Table III Further tests on the two inhibitory substances

Effect on Amoebae at Concentration $(\mu \mathrm{g} / \mathrm{ml})$

\begin{tabular}{llllllllll}
\hline 100 & 50 & 25 & 12.5 & 6 & 3 & 1.5 & 0.7 & 0.35 & 0.125
\end{tabular}

Hydroxystilbamidine

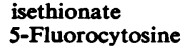

$\begin{array}{llllllllll}\text { AC } & \text { I } & \text { I } & \text { I } & \text { I } & \text { VI } & \text { VI } & \text { VI } & \text { VI } & \text { VI } \\ \text { AC } & \text { I } & \text { I } & \text { VI } & \text { NE } & \text { NE } & \text { NE } & - & - & -\end{array}$

Table IV Further tests on the two amoebicidal compounds 'Meaning of abbreviations as indicated at foot of Table II. each at concentrations of $100,80,60,40$, and 20 $\mu \mathrm{g} / \mathrm{ml}$, failed to show any potentiation of the inhibition indicated by the screen tests (Table II).

Sulphadiazine by itself was only feebly inhibitory against all the strains. However, Dr C. G. Cuthbertson (personal communication) found it to be effective in mouse protection tests with Hartmannella strains that he tested.

Amphotericin B was shown by Carter (1969), in laboratory studies, to be effective against Naegleria at a concentration of $0.075 \mu \mathrm{g} / \mathrm{ml}$. In my tests(TableIII) it was inhibitory to Hartmannella at $10 \mu \mathrm{g} / \mathrm{ml}$, but during prolonged incubation, which causes inactivation of the drug, survivors multiplied vigorously. The effect was greater than could be explained by the effect of the bile salt incorporated with the drug to effect solution.

Paromomycin, an aminoglycoside of the neomycin group, is effective against Entamoeba histolytica. Although it had a marked inhibitory effect on Hartmannella, the surviving amoebae were viable and multiplied rapidly when subcultured to medium free from paromomycin.

Hydroxystilbamidine isethionate is a diamidine with properties similar to stilbamidine but with considerably less human toxicity. It is used in the treatment of various protozoal and mycotie infections and may be used for a prolonged period (Snapper, Schneid, McVay, and Lieben, 1952). It may be incorporated into cells and remain active for long periods (Snapper, Schneid, Greenspan, and Lieben, 1950). Titration in all three media showed marked inhibition by as little as $0 \cdot 125 \mu \mathrm{g} / \mathrm{ml}$ for some strains (Table IV). A concentration of $100 \mu \mathrm{g} / \mathrm{ml}$ was amoebicidal but was toxic to the HeLa cells.

5-Fluorocytosine is a nucleotide analogue used in the treatment of infections due to cryptococci and candida, including cryptococcal meningitis (Watkins, Campbell, Gardner-Medwin, Ingham, and Murray, 1969). It was inhibitory at $12 \cdot 5$ $\mu \mathrm{g} / \mathrm{ml}$ and amoebicidal at $100 \mu \mathrm{g} / \mathrm{ml}$ (Table IV).

\section{Discussion}

Sensitivity testing of amoebae in vitro is much more difficult than tests on bacteria. The longer period of growth, the varying ability of different strains of the same species to grow in the same medium, and the ability of amoebae to encyst, all contribute to the difficulty. The correlation of sensitivity patterns shown by the three test systems used suggest that the techniques, as outlined here, probably give a fair indication of the sensitivity of these organisms and should be of use in testing other compounds.

The results of the tests in vitro reported here cannot be extrapolated to indicate therapeutic action; for example, amphotericin had no amoebicidal activity in my tests, but Apley et al (1970) have reported its use, with encouraging 
effect, for one proven and two probable cases of Naeglerial meningo-encephalitis.

Animal studies are in progress to determine whether the more active substances can protect mice against challenge infection of amoebae.

I wish to thank Dr M. S. Pereira for supplying five of the strains of amoebae, Dr J. Garrod of Roche Products Ltd, and Mr S. L. Squires of May \& Baker Ltd for the supply of drugs and much useful information, Boots Pure Drug Co. and Parke-Davis \& Co. for the supply of drugs, and Dr R. Blowers for help in the preparation of the typescript.

\section{References}

Adam, K. M. G. (1964). A comparative study of Hartmannellid amoebae. J. Protozool., 11, 423-30.

Apley, J., Clarke, S. K. R., Roome, A. P. C. H., Sandry, S. A., Saygi, G., Silk, B., and Warhurst, D. C. (1970). Primary amoebic meningoencephalitis in Britain. Brit. med. J., 1, 596-599.
Armed Forces Institute of Pathology (1968). Facultatively Patho genic Amoebae: A Survey on Nomenclature with a Biblios graphy, edited by J. L. Griffin. Armed Forces Institute of Pathology, Washington, DC.

Carter, R. F. (1968). Primary amoebic meningo-encephalitis: clinical, pathological and epidemiological features of
fatal cases. J. Path. Bact., 96, 1, 1-25.

Carter, R. F. (1969). Sensitivity to amphotericin B of a Naeglerto sp. isolated from a case of primary amoebic meninge encephalitis. J. clin. Path., 22, 470-474.

Neff, R. J. (1957). Purification, axenic cultivation and descriptio of a soil amoeba, Acanthamoeba sp. J. Protozool., 176-182.

Page, F. C. (1967a). Taxanomic criteria for Limax amoebae, witg descriptions of 3 new species of Hartmannella and 3 of Vahlkampfia. J. Protozool., 14, 499-521.

Page, F. C. (1967b). Re-definition of the genus Acanthamoeba with descriptions of three species. J. Protozool., 14, 709-724. -

Snapper, I., Schneid, B., Greenspan, E., and Lieben, F. (1950). Of the deposition of stilbamidine and 2-hydroxystilbamidine in cytoplasm and nuclei of different organs and tumours Bull. N.Y. Acad. Med., 26, 269-270.

Snapper, I., Schneid, B., McVay, L., and Lieben, F. (1952W Pharmacology and therapeutic value of diamidine derivios tives-particularly of 2-hydroxystilbamidine. Trans. N. Acad. Sci, 14, 269-271.

Sneath, P. H. A., and Stevens, M. (1967). A divided petri dish for use with multipoint inoculators. J. appl. Bact., 30, 495-49?

Warhurst, D. C., and Armstrong, J. A. (1968). Study of a small amoeba from mammalian cell cultures infarcted with ' $R$ yan Virus'. J. gen. Microbiol., 50, 207-215.

Watkins, J. S., Campbell, M. J., Gardner-Medwin, D., Inghank H. R., and Murray, I. G. (1969). Two cases of crypte coccal meningitis, one treated with 5-fluorocytosine. $\mathrm{Br}$ 审 med. J. 3, 29-31. 